\title{
PERCEPCIONES SOBRE LOS EFECTOS ECONÓMICOS, SOCIALES Y AMBIENTALES DEL MOTOTAXISMO EN MUNICIPIOS DEL DEPARTAMENTO DE BOLÍVAR (COLOMBIA)
}

Francisco Javier Maza Ávila Universidad de Cartagena Colombia

Rosario Blanco Bello Universidad de Cartagena Colombia

María Paula Fals Galezo Universidad de Cartagena Colombia 
Panorama Económico, Vol. 27 - No. 2 (Abril - Junio de 2019), pp. 349-369

Francisco Javier Maza Ávila $\quad$ Rosario Blanco Bello | María Paula Fals Galezo

JEL: R40, R41, R49

\title{
Percepciones sobre los efectos económicos, sociales y ambientales del mototaxismo en municipios del departamento de Bolívar (Colombia)
}

\section{Resumen}

Este artículo tiene como propósito develar las percepciones que construyen los habitantes de los municipios de Arjona, Turbaco, El Carmen De Bolívar y Magangué, del departamento de Bolívar, sobre los efectos económicos, sociales y ambientales de la dinámica del oficio de mototaxismo, así como la efectividad de las acciones llevadas a cabo por las autoridades municipales y departamentales para su control. La estrategia metodológica empleada fue de corte cualitativo, a fin de visibilizar las voces de los actores locales que interactúan y se relacionan con el territorio en su cotidianidad. Para ello se desarrollaron entrevistas semi-estructuradas, dirigidas a usuarios y no usuarios del servicio de mototaxis y transportadores públicos y privados, así como grupos focales, conformados por funcionarios públicos de las alcaldías municipales. Los resultados muestran que, si bien el oficio de mototaxismo ha facilitado la movilidad tanto de las personas como de la mercancía de productores y comerciantes locales (formales e informales), ha generado conflictos sobre quién o quienes pueden transportar personas y mercancías, dada la legitimación de un oficio informal e ilegal que genera consecuencias al servicio de transporte legal. El oficio también ha traído consigo efectos ambientales, reflejados en la calidad del aire, conservación del suelo rural, el atractivo visual del municipio y la audición de la población. Respecto a las medidas de regulación de oficio del mototaxismo, se infiere un bajo conocimiento de ellas. Quienes indican conocerlas, consideran son poco efectivas, puesto que son transitorias y, en algunos casos, carecen de planificación.

Palabras clave: Oficio de mototaxismo, Percepciones, Efectos económicos, Efectos sociales, Efectos ambientales, Departamento de Bolívar.

\section{Perceptions sur les effets économiques, sociaux et environnementaux du taxisme motocycliste dans les municipalités du département de Bolívar (Colombie)}

\section{Résumé}

Le but de cet article est de révéler les perceptions que les habitants des municipalités d'Arjona, Turbaco, El Carmen De Bolívar et Magangué, du département de Bolívar, construisent. Accent sur les effets économiques, sociaux et environnementaux de la dynamique du commerce du mototaxisme, ainsi que sur l'efficacité des actions menées par les autorités communales et départementales pour son contrôle. La stratégie méthodologique utilisée était de nature qualitative, afin de rendre visible la voix des acteurs locaux qui interagissent et se rapportent au territoire dans leur vie quotidienne. Pour cela, des entretiens semi-structurés ont été organisés, à destination des usagers et non-usagers du service de taxi moto et des transporteurs publics et privés, ainsi que des focus groups, composés de fonctionnaires des maires de la commune. Les résultats montrent que, bien que le commerce du mototaxisme ait facilité la mobilité des personnes et des marchandises des producteurs et des commerçants locaux (formels et informels), il a généré des conflits sur qui ou qui peut transporter des personnes et des marchandises, étant donné la légitimation d'une commerce illégal qui génère des conséquences pour le service de transport légal. Le commerce à également des effets environnementaux, qui se produit dans la qualité de l'air, la conservation du sol rural, l'attrait visuel de la municipalité et l'audition de la population. En ce qui concerne les mesures réglementaires d'office pour le taxisme motocycliste, une faible connaissance a été conclue. Ceux qui indiquent une certaine connaissance, ils les considèrent comme inefficaces, car ils sont transitoires et, dans certains cas, manquent de planification.

Mots-clés: Bureau de mototaxisme, Perceptions, Effets économiques, Effets sociaux, Effets environnementaux, Département de Bolívar.

\section{Perceptions on the economic, social and environmental effects of motorcycle taxism in municipalities of the department of Bolívar (Colombia)}

\begin{abstract}
The purpose of this article is to reveal the perceptions that the inhabitants of the municipalities of Arjona, Turbaco, El Carmen De Bolívar and Magangué, of the department of Bolívar, construct. Emphasis on the economic, social and environmental effects of the dynamics of the mototaxism trade, as well as the effectiveness of the actions carried out by the municipal and departmental authorities for its control. The methodological strategy used was of a qualitative nature, in order to make visible the voices of local actors who interact and relate to the territory in their daily lives. For this, semi-structured interviews were held, aimed at users and non-users of the motorcycle taxi service and public and private transporters, as well as focus groups, made up of public officials from the municipal mayors. The results show that, although the mototaxism trade has facilitated the mobility of both people and merchandise from local producers and merchants (formal and informal), it has generated conflicts over who or who can transport people and goods, given the legitimization of an informal and illegal trade that generates consequences for the legal transport service. The trade has also brought with it environmental effects, reflected in air quality, conservation of rural soil, the visual appeal of the municipality and the hearing of the population. Regarding the ex officio regulatory measures for motorcycle taxism, low knowledge was concluded. Those who indicate some knowledge, they consider them to be ineffective, since they are transitory and, in some cases, lack planning.
\end{abstract}

Keywords: Mototaxism office, Perceptions, Economic effects, Social effects, Environmental effects, Department of Bolívar. 


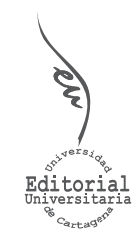

Artículo de Investigación

\title{
Percepciones sobre los efectos económicos, sociales y ambientales del mototaxismo en municipios del departamento de Bolívar (Colombia) '
}

\author{
Francisco Javier Maza Ávila* \\ Universidad de Cartagena, Colombia \\ Rosario Blanco Bello \\ Universidad de Cartagena, Colombia \\ María Paula Fals Galezo \\ Universidad de Cartagena, Colombia
}

INFORMACIÓN DEL ARTÍCULO

Recepción del artículo: 27/09/2018

Concepto de evaluación: 30/11/2018

Aceptación del artículo: 17/01/2019

\section{INTRODUCCIÓN}

Los municipios de Arjona, El Carmen de Bolívar, Magangué y Turbaco, son todos pertenecientes al departamento de Bolívar. El municipio de Turbaco se ubica en la Zona de Desarrollo Económico y Social -Zodes- ${ }^{2}$ Dique. Cuenta con una extensión de $196 \mathrm{Km} 2$ y está a una distancia 9,9 Km de la capital del departamento de Bolívar (Alcaldía Municipal de Turbaco, 2016). El municipio de Arjona también se encuentra localizado al norte del departamento, en la Zodes Dique, limitando al norte con el municipio de Turbaco. Cuenta con una extensión territorial de $616,5 \mathrm{Km} 2$ y se encuentra a una distancia de $33 \mathrm{~km}$ de la capital del Departamento (Alcaldía Municipal de Arjona, 2016). El Carmen de Bolívar, por su parte, es un municipio ubicado en el sistema orográfico de la Serranía de San Jacinto, muy cerca del litoral Caribe colombiano y perteneciente Los Montes de María (Alcaldía Municipal El Carmen de Bolivar, 2016), una de las regiones tristemente célebres por ser el epicentro del conflicto armado en la región Caribe colombiana (Maza \& Perez 2015). Magangué, por último, es un municipio colombiano localizado en todo el centro del departamento y a orillas del río Magdalena. Se encuentra ubicada entre la Depresión Momposina y la Mojana Bolivarense. Esta ciudad bolivarense es conocida como "La capital

\footnotetext{
*Autor de correspondencia: fmazaa@unicartagena.edu.co

1. Este artículo se desprende de la investigación titulada "El transporte informal en los municipios de Arjona, Turbaco, el Carmen de Bolívar y Magangué”, elaborada por el Instituto Internacional de Estudios del Caribe de la Universidad de Cartagena para el Centro de Observación e Investigación Social de Bolívar -COISBOL- de la Gobernación de Bolívar.

2. En el 2001, y con el objetivo de lograr una adecuada articulación territorial de la población y de los distintos sectores productivos, así como una coordinación y planeación que garantizara su crecimiento y desarrollo, las autoridades del departamento de Bolívar, mediante acto legal, subdividieron el departamento en seis Zonas de Desarrollo Económico y Social -Zodes-, abarcando cada una de ellas a varios municipios.
} 
de los ríos", ya que en este lugar del país desembocan los ríos Cauca y San Jorge en el Magdalena. Magangué también es capital -de facto- de la Región de La Mojana (Alcaldía Municipal de Magangué, 2016).

Según proyecciones para el año 2017 elaboradas por el Departamento Administrativo Nacional de Estadísticas (DANE), la población total de los municipios de Turbaco, Arjona, El Carmen de Bolívar y Magangué es de 350.335 personas, equivalente al 16,3\% de la población del departamento de Bolívar y el 0,71\% de la población total de Colombia. Con el 35,4\% de la población total, Magangué se constituye en el municipio más poblado, seguido de El Carmen de Bolívar (22\%), Arjona (21,5\%) y Turbaco (21,2\%). Estos se caracterizan por tener una densidad poblacional para el año 2017 de 173 hab/km2, muy superior a los promedios departamentales y nacionales (Tabla 1).

El análisis de las condiciones económicas, sociales e institucionales de los municipios de Arjona, Turbaco, El Carmen de Bolívar y Magangué permiten inferir su alta dependencia de los recursos transferidos por el Estado, en especial, los del Sistema General de Participaciones (Ley 715 de 2001), para atender la seguridad social en salud, educación y agua potable y saneamiento básico de su población. Además, muestra una pobre capacidad de gestión integral, vista desde la bajo cumplimiento de las políticas económicas y sociales planteadas en su plan de desarrollo, la ineficiencia en la gestión de los recursos del Sistema General de Participación, y su bajo desempeño fiscal, lo que retrasa la posibilidad de lograr un desarrollo económico sostenible, en bienestar de sus habitantes (Maza et al., 2017)

Tabla 1.

Fundación, distancia de Cartagena de Indias y extensión de los municipios de Turbaco, Arjona, El Carmen de Bolívar y Magangué

\begin{tabular}{|c|c|c|c|c|}
\hline MUNICIPIO & TURBACO & ARJONA & $\begin{array}{l}\text { EL CARMEN } \\
\text { DE BOLIIVAR }\end{array}$ & MAGANGUÉ \\
\hline CÓDIGO MUNICIPAL & 13836 & 13052 & 13244 & 13430 \\
\hline SUBREGIÓN & $\begin{array}{l}\text { DIQUE } \\
\text { BOLIVARENSE }\end{array}$ & $\begin{array}{l}\text { DIQUE } \\
\text { BOLIVARENSE }\end{array}$ & $\begin{array}{l}\text { MONTES } \\
\text { DE MARÍA }\end{array}$ & $\begin{array}{l}\text { MOJANA } \\
\text { BOLIVARENSE }\end{array}$ \\
\hline $\begin{array}{l}\text { CREACIÓN } \\
\text { COMO MUNICIPIO }\end{array}$ & 1510 & 1770 & 1857 & 1610 \\
\hline SUPERFICIE KM2 & 196 & 566 & 900 & 1102 \\
\hline $\begin{array}{l}\text { DISTANCIA DE CARTAGENA } \\
\text { DE INDIAS KM2 }\end{array}$ & 16,19 & 35,18 & 114 & 216 \\
\hline $\begin{array}{l}\text { ÁREA DEL MUNICIPIO/ } \\
\text { ÁREA DEPARTAMENTAL }\end{array}$ & $0,8 \%$ & $2,2 \%$ & $3,5 \%$ & $4,2 \%$ \\
\hline POBLACIÓN & 74.209 & 75.271 & 76.949 & 123.906 \\
\hline DENSIDAD POBLACIONAL & 368,20 & 128,12 & 83,506 & 112,28 \\
\hline CATEGORÍA MUNICIPAL & 5 & 6 & 6 & 6 \\
\hline ENTORNO DE DESARROLLO & INTERMEDIO & INTERMEDIO & INTERMEDIO & INTERMEDIO \\
\hline
\end{tabular}

Fuente: Instituto Geográfico Agustín Codazzi- Colombia; Departamento Nacional de Planeación -DNP- 
La insuficiencia de la descentralización administrativa y de la baja capacidad institucional para fortalecer el aparato productivo de la región que promueva un desarrollo local sostenible, se traducen en el bajo acceso a servicios sociales prioritarios -salud, educación, servicios público, entre otros- y profundos niveles de pobreza y miseria, reflejados en el notable desequilibrio en términos de pobreza entre la zona urbana y la zona rural; de acuerdo con el Índice de Pobreza Multidimensional -IPM-, desarrollado por el Oxford Poverty \& Human Development Initiative -OPHI- (Angulo \& Diaz, 2011), en la zona rural existen un mayor número de personas que habitan en hogares multidimensionales pobres que en la zona urbana; la incidencia promedio de la zona urbana de pobreza multidimensional es de $77 \%$ y para la zona rural es del $86 \%$, alrededor de 10 puntos porcentuales de diferencia. Este contexto, caracterizado por desigualdades e inequidades sociales a nivel municipal, ha contribuido al surgimiento y la persistencia de una gran variedad de oficios informales, en respuesta a la necesidad de proveer ingresos a los hogares por parte de las familias con las más bajas posibilidades de integrarse al mercado laboral formal. Entre ellos, ha tomado mayor fuerza el oficio de mototaxismo, como un medio de transporte alternativo ante la ausencia de un transporte público formal, principalmente en las zonas de difícil acceso.

El transporte informal de pasajeros en motocicletas en Colombia -conocido comúnmente en la región Caribe como "mototaxi"-, nació a principios de la década de los 90's como respuesta entre los desempleados de las zonas marginales del departamento de Córdoba, propietarios de motocicletas (Brieva et al., 2011). En la actualidad, el servicio de mototaxi es el medio de transporte informal más utilizado por diversas poblaciones del país -es común en, al menos, 26 de los 32 departamentos de Colombia-, con especial énfasis en los departamentos de la región Caribe colombiana, y se ha convertido en el medio de supervivencia laboral para personas que, en su mayoría, son de escasos recursos (Castillo et al., 2013). Si bien no existen cifras concretas, se estima que Bolívar es uno de los departamentos de la región Caribe con la mayor presencia de este servicio de transporte informal en sus municipios. Además de las altas tasas de desocupación que convierten al mototaxismo en una importante -si no la única- fuente de ingresos para quienes ejercen el oficio, otras razones que pueden explicar su crecimiento es la escasa o nula presencia de un servicio de transporte formal, a nivel municipal, que satisfaga las necesidades de movilidad entre los habitantes, además de la importante aceptación que tiene entre sus usuarios del servicio (Maza et al., 2017).

Aunque el mototaxismo parece minimizar el problema de movilidad en los municipios donde este servicio informal detransporte se desarrolla, seconstituyeen una problemática social y de salud pública; el incremento de motocicletas dedicadas a esta labor en las vías genera un mayor riesgo para quienes se ocupan en este oficio. Un mayor número de accidentes, enfermedades causadas por la exposición constante a los cambios climáticos e inadecuadas condiciones para el ejercicio de su labor conducen a un desequilibrio en el estado de salud de la persona y, por ende, a una mala calidad de vida (Castillo et al., 2013). Por sólo mencionar algunos datos, en materia de accidentalidad, según las estadísticas del Observatorio Nacional de Seguridad Vial, para el año 2018, el 74\% de las muertes y el 65,38\% de los lesionados en accidentes de tránsito en los municipios de Arjona, El Carmen de Bolívar, Magangué y Turbaco involucran una motocicleta. Dichas cifras están muy por encima de las registradas tanto a nivel del departamento de Bolívar (64,61\% vs 59,32\%) como nacional (50,48\% vs 56,03\%). La situación anterior advierte que el mototaxismo es un tema de contrastes: por un lado, es una alternativa de subsistencia y minimización del déficit de transporte público, pero por otra parte es fuente de problemáticas sociales. 
Teniendo en cuenta el papel que juega el mototaxismo en la región, así como los riesgos que este oficio supone tanto para quien lo ejerce, como para los diversos autores relacionados, este artículo tiene como propósito develar las percepciones que construyen los habitantes de los municipios de Arjona, Turbaco, El Carmen De Bolívar y Magangué, del departamento de Bolívar -entre usuarios, no usuarios, funcionarios públicos municipales y transportadores locales- sobre los efectos económicos, sociales y ambientales de la dinámica del oficio de mototaxismo, así como la efectividad de las acciones llevadas a cabo por las autoridades municipales y departamentales para su control. Los resultados de este artículo pretenden servir como insumo para el diseño de lineamientos para una política pública que contribuya al tratamiento de la problemática sobre este medio de transporte no-formal.

\section{ESTADO DEL ARTE}

La alta demanda de transportes alternativos ha ocasionado transformaciones en la movilidad de las ciudades, a nivel mundial. En general, las preferencias por opciones diferentes al transporte público autorizado han estado ligadas al bajo costo, rapidez, ahorro de tiempo, así como a aspectos de índole cultural que, en definitiva, parecen estar por encima de la exposición a graves riesgos por quienes emplean dichos medios de transporte (Chavis \& Daganzo, 2013). Lo anterior ha motivado el diseño e implementación de políticas y normas encaminadas tanto para prohibir el uso de motocicletas de dos y tres ruedas, o de vehículos particulares para el transporte público, como para estimular el uso de otros medios de transporte, tales como las bicicletas, con diferentes niveles de efectividad entre países (Guillen \& Ishida, 2004; Simon et al., 2016).

La revisión de la literatura muestra que en África, Asia y Latinoamérica, las alternativas diferentes al transporte público autorizado tienen como común denominador el uso de motocicletas, consideradas ilegales para ejercer dicho oficio, dado los riesgos que trae consigo su uso, principalmente para el conductor o "mototaxista", tales como la exposición a condiciones perjudiciales para la salud y/o el incremento del riesgo de accidentalidad por el ejercicio del oficio, la falta de uso del equipamiento y la violación de normas de tránsito. Para el caso de África, las investigaciones identificadas tratan sobre la exposición a riesgos que puedan afectar la salud de los mototaxistas (Avogbe et al., 2011), así como al desarrollo y análisis de la estructura de los sistemas informales de tránsito (Chavis \& Daganzo, 2013). A nivel latinoamericano, las investigaciones centran su interés en identificar las causas que motivan el uso de motocicletas para el transporte urbano (Hagen et al., 2016), así como a la prevalencia de lesiones faciales y del absentismo en mototaxistas que son víctimas de accidentes (Barbosa et al., 2014), además del abordaje de la influencia de los elementos de protección personal en las lesiones que puede causarle un accidente al motociclista (Cini et al., 2014).

En Colombia, el transporte público se ha caracterizado por su poca diversificación frente a la necesidad de opciones más eficientes y de bajo costo, lo que ha arrojado como resultado la alta demanda de alternativas informales e ilegales como el mototaxismo y, por lo tanto, el incremento de ofertantes, normalmente conductores inexpertos y con bajo conocimiento de las normas de tránsito. Además, el fenómeno del mototaxismo se ha considerado como una alternativa de transporte socio-culturalmente aceptada, pese a ser ilegal y al riesgo para la salud de los usuarios. De hecho, para varias ciudades de la región Caribe colombiana 
resulta un estímulo para la economía, pese a las condiciones de vulnerabilidad para quienes ejercen el oficio, en materia de afiliación a la Seguridad Social, el acceso a beneficios fundamentales de un trabajo digno, y los riesgos físicos y ergonómicos. La revisión de la literatura arrojó estudios centrados en las causas que motivan el ejercicio del oficio y su coincidencia en los aspectos sociales y económicos de las ciudades donde tiene presencia (Castillo Osorio, 2010; Espinosa et al., 2008); otros estudios analizan las condiciones de trabajo y salud de los mototaxistas (Castillo Avila et al., 2013; Hinestroza-filigrana \& Toro-mayor, 2015), mientras que otras investigaciones analizan los niveles de accidentalidad y las discapacidades generadas (Aldana Oyola, 2013; Herazo B. et al., 2011; Jimenez et al., 2015).

Pese a los avances en las líneas de investigación referidas a efectos en la salud, accidentalidad, lesiones de los mototaxistas y la intervención del oficio en las dinámicas urbanas, son escasos los estudios que arrojan información sobre las percepciones que han construido los usuarios, no usuarios, transportadores locales y entidades gubernamentales sobre los efectos que ejerce el oficio de mototaxismo en sus territorios, propósito central de este artículo de investigación. Por otro lado, prevalece el enfoque de investigación cualitativo, por cuanto es oportuno profundizar en la dimensión subjetiva de la realidad social de los actores alrededor del oficio de mototaxismo.

\section{METODOLOGÍA}

La estrategia metodológica empleada en esta investigación fue corte cualitativa, de modo que posibilitara la reivindicación de las realidades subjetivas como objeto de conocimiento, develando las singularidades construidas por los actores locales, desde su cotidianidad, sobre su municipio. En este sentido, y según Sandoval Casilimas (1996, p. 93), se concibe la investigación cualitativa como: a) La recuperación de la subjetividad como espacio de construcción de la vida humana; b) La reivindicación de la vida cotidiana como escenario básico para comprender la realidad socio-cultural, y c) La intersubjetividad y el consenso, como vehículos para acceder al conocimiento válido de la realidad humana. Desde este punto de vista, se privilegian las voces de los actores locales de los municipios de Arjona, El Carmen de Bolívar, Magangué y Turbaco, en tanto agentes activos en la identificación del estado actual del oficio de mototaxi y en la planeación de alternativas de solución para tratar la problemática sobre este medio de transporte informal en su municipio.

El estudio de las percepciones implica tener "en cuenta tres componentes interdependientes (...): el atributivo (que conlleva la categorización de lo percibido); el afectivo (que supone una apreciación o valoración de lo percibido); y el expectativo (que atribuye significaciones prescriptivas a los distintos elementos del entorno percibido" (Gómez, H.: 1982, p. 131. Citado en González, E.: 2008, p. 122). En otras palabras, la percepción es un proceso mediante el cual las personas identifican un objeto, lo valoran y proyectan expectativas frente a éste. En esta oportunidad las percepciones son las construcciones elaboradas por los ciudadanos en torno al oficio de mototaxi que se ejerce en los municipios donde residen.

Las técnicas cualitativas utilizadas para la recolección de información fueron la entrevista semi-estructurada y los grupos focales, dirigidas a los grupos de interés identificados -funcionarios públicos, usuarios y no usuarios del servicio de mototaxi, y transportadores 
formales-. A través de la entrevistas individualizadas y grupales, entendidas como "una conversación con un propósito o intencionalidad (...) fijada a partir de unos objetivos de investigación" (Binggan y Moore, 1973; en Rubio \& Varas, 2004); por su parte, el grupo focal se realizó con el propósito de recuperar las creencias, actitudes y sentimientos provocados en la interacción entre los participantes sobre una temática propuesta por el investigador, a fin de obtener una multiplicidad de perspectivas en el grupo (Escobar \& Bonilla Jimenez, 2009). El uso de estas técnicas, facilitó obtener información primaria relevante, que posibilitó acercarse a la realidad de los municipios, desde de los discursos de los actores locales.

Las fuentes información primaria corresponden a los actores locales del municipio, representados en funcionarios públicos, usuarios y no usuarios del servicio de mototaxi, y transportadores formales. Los criterios de selección de la muestra no probabilística fueron los siguientes: personas mayores de 18 años de edad, que residan en los municipios focalizados y que tuvieran la disposición a participar en la investigación. Asimismo, el número de entrevistas aplicadas dependió de la saturación de las categorías de análisis (Sandoval Casilimas, 1996). La relación entre la técnica cualitativa utilizada y los grupos de interés -que se constituyen en la fuente primaria de información- se describe en la siguiente tabla:

Tabla 2.

Técnica cualitativa vs grupos de interés

\begin{tabular}{|c|c|c|}
\hline TÉCNICA CUALITATIVA & $\begin{array}{l}\text { FUENTE PRIMARIA } \\
\text { DE INFORMACIÓN }\end{array}$ & NÚMERO DE ENTREVISTAS \\
\hline $\begin{array}{l}\text { ENTREVISTA } \\
\text { SEMI-ESTRUCTURADA }\end{array}$ & $\begin{array}{l}\text { Usuarios del servicio de mototaxi. } \\
\text { No usuarios del servicio de mototaxi. } \\
\text { Transportadores públicos y/ } \\
\text { privados que ofrecen servicio } \\
\text { de transporte público. }\end{array}$ & 76 entrevistas \\
\hline GRUPO FOCAL & $\begin{array}{l}\text { Funcionarios públicos de las alcaldías } \\
\text { municipales (Secretaría de Tránsito y } \\
\text { Transporte de Bolívar, Secretaria de } \\
\text { gobierno, Secretaría de Planeación y } \\
\text { Secretaría de salud) }\end{array}$ & $\begin{array}{l}5 \text { entrevistas y } \\
2 \text { grupos focales. }\end{array}$ \\
\hline
\end{tabular}

Fuente: Elaboración propia

\section{RESULTADOS}

Las percepciones sociales son el resultado de un proceso de interacción social, que consta del conocimiento de los otros, el reconocimiento de emociones, de la formación de impresiones y atribuciones, la cuales están condicionadas por las motivaciones, necesidades, valores, aprendizajes y experiencias de las personas (Arias Castilla, 2006). El estudio de la percepción social trasciende la mirada sensorial, de corte biológico y físico, y pasivo, para orientar su enfoque hacia las categorizaciones y significaciones que se construyen desde lo relacional; en este orden de ideas, la percepción es biocultural 
porque, por un lado, depende de los estímulos físicos y sensaciones involucrados y, por otro lado, de la selección y organización de dichos estímulos y sensaciones (Vargas Melgarejo, 1994).

En esta sección se muestran los resultados de las percepciones sobre los efectos económicos, sociales y ambientales del oficio de mototaxismo en Arjona, El Carmen de Bolívar, Magangué y Turbaco, así como la efectividad de las acciones llevadas a cabo por las autoridades municipales y departamentales para su control.

\section{Percepciones sobre efectos económicos del oficio de mototaxismo}

En este apartado, se describen los efectos del oficio de mototaxi sobre el transporte formal e informal -colectivo, bicitaxis- que tiene presencia en los municipios, así como en la dinámica económica de los pequeños productores, comerciantes y vendedores ambulantes.

\section{Efectos del mototaxismo en el transporte formal e informal}

La preferencia de la población por el servicio de mototaxi obedece a su rapidez frente al trasporte convencional, prestan un servicio de puerta a puerta y son a bajo costo. Estas cualidades del servicio generan conflictos sobre quién o quienes pueden transportar personas, teniendo en cuenta que se ha legitimado una práctica ilegal que genera unas consecuencias en el servicio de transporte legal. En algunos municipios, el transporte informal sólo es usado en los trayectos intramunicipales, dado que no hay presencia de un transporte legal que interconecte los barrios o la cabecera municipal con la zona rural. En este sentido, el uso de las motos cobra importancia, dada la inexistencia de otras alternativas de transporte formal.

"Pues eso, eso es dependiendo de pronto en un pueblo como éstos, pues no afecte en nada a otros gremios de transportadores porque no los hay, no hay taxistas, no hay buses de servicio público, pero en ciudades grandes si perjudica va a afectar de pronto el Transcribe ahí en Cartagena los buses de servicio público y los taxistas si los va afectar". Entrevista a Usuario5, Arjona.

Los efectos negativos del oficio de mototaxismo en el servicio de transporte formal se refleja, principalmente, en la inseguridad vial teniendo en cuenta que algunos transportadores afirman que la congestión vial provocada por la cantidad de motos, dificulta la circulación y el estacionamiento de los de buses municipales.

"A nosotros nos afecta bastante en cuanto al producido, y la congestión vial que aquí hay en Turbaco, ahora mismo, no se puede manejar, por qué, porque hay tanta moto, que en cualquier parte encuentra una moto parqueada". Entrevista a Transporadores3, Turbaco.

No obstante, los entrevistados refieren que los mototaxistas facilitan el transbordo de los pasajeros desde lugar de residencia -o partida-, hacia la estación de buses intermunicipales que viajan hacia Cartagena o Turbaco.

"Pues yo no creo que los afecte al contrario los ayuda, porque en el momento de transbordar están ellos ahí para transportarlos hasta la cabecera municipal, (...) hasta el centro de Arjona porque los buses muchas veces lo dejan a uno en una 
esquina, quizás uno tenga que caminar (...) pues facilitan el transporte”. Entrevista NoUsuario5, Arjona.

En el caso de Magangué, algunas personas prefieren usar las motos en lugar de taxis y busetas, porque la prestación del servicio de transporte formal es deficiente y tardío; además, no circulan en todos los barrios el municipio. Sin embargo, las opiniones sobre las afectaciones del servicio de mototaxi sobre el transporte formal están divididas, pues algunos expresan que cada servicio tiene sus clientes, y que no tendría por qué generar efectos negativos. Otros, por su parte, sostienen que la preferencia por la moto ha desplazado el uso de otros medios de transporte reduciendo, sustancialmente, la presencia del transporte informal, tales como los colectivos en jeeps o camionetas y las bicitaxis, que usualmente facilitaban la conexión interna, pero también entre la cabecera municipal y la zona rural, ante la ausencia un transporte público formal en los municipios.

"Yo creo que ni los afecta ni los beneficia. Porque una carrera de taxi la mínima aquí en Magangué está en 4mil pesos, algunas personas, no todos tienen para pagar eso en una carrera. Sí hay 10, 15 taxi es mucho y siempre están parados en las puertas de los supermercados, tampoco hay un número donde puedas llamar a pedir un taxi de urgencia, entonces creo que el medio más rápido es la moto. Entonces por eso creo que ni los afecta, ni los beneficia, todo aquél que quiere un taxi lo puede pedir, puede tener el número del conductor y puede esperar y el que no puede ser en otro. Entonces para mí ni los afecta ni los beneficia. Cada quien tiene su plaza, su clientela". Entrevista NoUsuario5M, Magangué.

“(..) los Jeeps que antes viajaban a los corregimientos, a la Alta Montaña se han visto muy afectados porque, una persona tiene que esperar 20 minutos, media hora, hasta una hora, que se llene un Jeeps, para ir hasta cualquier corregimiento, sea Caracolí, Cansona, o Macayepo (...), entonces prefieren pagar $\$ 5000$ una moto para que lo lleve (...)" Entrevista Funcionario1, Carmen de Bolívar.

\section{Efectos del mototaxismo en los pequeños productores y comerciantes.}

El mototaxismo ha beneficiado a los pequeños productores que residen o trabajan en las zonas rurales, teniendo en cuenta que facilita el acceso a sus parcelas y, además, prestan el servicio de transporte de carga, por ejemplo, de maíz, yuca, ñame, entre otros. En este sentido, se puede afirmar que este servicio ha desplazado la utilización de mulas, burros y caballos, que anteriormente eran adiestrados para este tipo de oficios de carga, reduciendo así el tiempo y los costos del traslado, además de los costos asociados al sostenimiento de dichos animales.

"(...) los animales de carga que antes utilizaban ya no se utiliza ahora los pequeños productores incluso tiene dentro de sus parcelas cuenta con una motocicleta o por lo por lo menos con algún conocido que la tenga y a través de estas hacen su desplazamiento y de su producto. Entrevista a Funcionarios1, Turbaco

Respecto los efectos del mototaxismo en los comerciantes, se reconoce que este servicio de mototaxi permite el ingreso y la salida de los clientes de establecimientos comerciales, facilitando la rotación de usuarios y la prestación de un servicio oportuno, de esta forma, ambos reciben beneficios. Asimismo, los mototaxis son contratados por los 
comerciantes para transportar los productos que deben surtir en sus establecimientos.

"Dinamiza la economía, si mueves población mueves dinero (...) Incluso los mismos almacenes los parquean en las puertas y les dan uniformes, claro le financia los chalecos, porque les conviene que les trae a los clientes". Grupo Focal funcionarios1, Carmen de Bolívar.

No obstante, se afirma que los comerciantes están expuestos a ser víctimas de actos delictivos, pues la aglomeración de motocicletas en las afueras de los locales, no permite identificar con facilidad quien ejerce como mototaxista, o como delincuente.

"la inseguridad, (...) cuándo se ponen en las puertas de enfrente de los comerciantes, no saben cuáles son las intenciones de ellos [mototaxistas], si se van a llevar un cliente de verdad, verdad, hay veces Entrevista a Transportares4, Turbaco.

Por otro lado, la ocupación por parte de los mototaxistas del espacio previsto para el cargue y descargue de mercancías en los locales comerciales, obstaculiza el fácil acceso a éstos.

\section{Efectos del mototaxismo en los pequeños productores y comerciantes.}

La relación entre los mototaxistas y vendedores ambulantes es de mutuo beneficio; por un lado, establecen una relación comercial, en cuanto a la venta y compra de comida, por el otro, utilizan como un medio de transporte para llegar a sus puestos de trabajo. En este mismo sentido, algunos entrevistados afirman que estas transacciones entre vendedores ambulantes y mototaxistas congestionan las vías, a causa de la ocupación informal del espacio público, lo que termina limitando la movilidad.

"Ya ahí de pronto se genera como que un caos por hay veces que se acumula mucho mototaxi en una parte, en una calle que de pronto congestiona la vía y los vendedores a veces no tienen la capacidad como que tener un espacio para vender sus productos, entonces congestiona la vía" Entrevista a Usuario4, Magangué.

"(...) por lo menos hay unos vendedores ambulantes que le venden específicamente a los mototaxis, el tema de desayuno, del almuerzo de pronto si se prohíbe el mototaxismo, me imagino que esas personas se van a ver afectadas. . Entrevista a Funcionario2, Magangué.

\section{Percepciones sobre efectos sociales del oficio de mototaxismo}

El oficio de mototaxismo ha intervenido notablemente en la transformación de las dinámicas sociales, culturales, económicas y políticas de los municipios. En este apartado se describen las percepciones que los actores locales han construido con relación a los efectos sociales del mototaxismo en sus municipios, enfatizando en su incidencia en la seguridad ciudadana, la convivencia y el orden público.

\section{Efectos en la seguridad ciudadana}

La seguridad ciudadana, vista desde los derechos humanos, obedece a "las condiciones que generan un contexto que minimiza los riesgos y potencia el tejido social para que las 
personas, en tanto ciudadanos sujetos de derechos, puedan desarrollarse de acuerdo a los proyectos de vida que han definido (Ojeda, 2005). Uno de los fenómenos del contexto que, de acuerdo a los entrevistados, condiciona la percepción de seguridad ciudadana, es el oficio del mototaxismo; en este sentido, es concebido como un oficio que limita la libertad humana, en tanto se ha asociado con actos delictivos que atentan contra la seguridad y el bienestar de las personas, y que han llevado a la pérdida de confianza en el otro. De esta forma, aun cuando el servicio es utilizado, ante la inexistencia de otros, se prefiere que quien ejerza la actividad sea alguien conocido.

"Sí yo creo que está afectando la seguridad del municipio, o sea uno aquí no se atreve a coger cualquier mototaxi, tiene que ser conocido, o sea no se atreve (...) puede ser algún vándalo" Entrevista a Usuario7, Carmen de Bolívar.

"Bueno he podríamos decir que algunos motociclistas realizan actividades ilegales, la mayoría pues realiza el servicio de transporte Pero hay algunos pues que realizan actividades ilegales e ilícitas, no solamente lo que tiene que ver con hurtos si no también con micro tráfico, con extorsiones, con algunos delitos sexuales, que también desafortunadamente se han cometido acá en nuestra cabecera municipal, entonces son situaciones que afectan a los que habitamos en este municipio" Entrevista Funcionario 1, Turbaco.

De este modo, se reafirma la desconfianza e inseguridad referido a quién ejerce el oficio y cuál es la intencionalidad de usar una motocicleta. Lo anterior conlleva a la fragmentación de las relaciones sociales armónicas, teniendo en cuenta que el miedo y la incertidumbre de ser víctima de un acto delictivo protagonizado por una persona en moto, conduce a que los actores locales vivan con una angustia constante.

"Claro que sí, lo afecta, lo afecta mucho, eso la verdad es que yo, yo digo que eso, las motos han acabado como con la paz, con la paz y con la tranquilidad del pueblo, porque uno sale y es con miedo que uno no pueda atravesar las calles, uno no pueda atravesar de un lado hacia otro porque tiene que estar ipila! con la moto porque se lo llevan a uno" (...) y también eso ha generado más este atracos, robos, de todo, este si más violencia porque aja matan". Entrevista NoUsuario2, Turbaco.

Ahora bien, no sólo se ha normalizado el estar expuestos a actos delictivos, sino que se han construido estereotipos que relacionan los actos delictivos con quienes que ejercen este oficio. Dicha situación se ha perpetuado por el poco o nulo control de las autoridades pertinentes para dar una solución viable al problema de inseguridad, a nivel municipal.

“(...) la autoridad es quien le competen para montar los frentes de seguridad y aquí la autoridad prácticamente es nula, aparte, tú llamas al cuadrante y aparecen cuando en verdad ya no tienen nada que hacer (...) considero que nosotros, la comunidad estamos huérfano de autoridad, (...) yo estoy seguro que la policía, los agentes saben, quiénes son los que a través de mototaxismo delinque, sabe quiénes son los que atracan, Entrevista Transportador4, Turbaco. 
Esimportante resaltar quealgunos entrevistados manifiestan que, independientemente del mototaxismo, hay un problema de seguridad ciudadana, reflejado en los actos delictivos y la violencia urbana que no son orquestados desde una motocicleta. Tanto así, que los mismos mototaxistas han sido víctimas de hurto. Por lo tanto, la prohibición de esta actividad no garantiza la erradicación de la inseguridad.

"No la verdad no yo, yo el mal siempre existe si igual quitan el mototaxismo igual se van a seguir dando los casos de, de atraco o fleteo que son los que más se originan dentro de las motos entonces eso no es lo que va lo que va realmente a subsanar que se dejen de dar esos casos, el mototaxismo si bien no es legal pienso que han generado muchas cosas más buenas que negativas" EUsuario9A

\section{Efectos en la convivencia ciudadana en escenarios colectivos}

La convivencia ciudadana se expresa en el "reconocimiento y respeto por la diferencia y la diversidad, en un plano de libertad, de igualdad ante la ley y de solidaridad, dentro del marco de la vida en sociedad" (Burbano, 2009); es decir, se basa en las relaciones de respeto y solidaridad que consolidan los lazos comunitarios y fraternos. De este modo, a continuación, se muestran las percepciones sobre la relación mototaxista-usuario, durante la prestación del servicio, y la relación mototaxista -otros conductores.

La valoración de la prestación del servicio de los mototaxis es muy variada. Va desde bueno a regular y deficiente. Las razones por las cuales afirman que es un buen servicio, obedecen al trato amable y colaborador, y a su disposición de llevarlos a cualquier lugar, sin reparos.

"La verdad es que es bueno, hay unos que son groseros, no te voy a decir que a mí no me ha tocado uno, me han tocado unos groseros, pero aja hay otros que son muy amables, hay algunos que no ayudan ni a bajar las bolsas como hay otros que si te entran hasta la terraza, te las ponen allá muy amable, o sea hay es que escogerlo" (Depende del mototaxi) "si". Entrevista a Usuario2, Turbaco.

Sin embargo, la gran mayoría de los entrevistados considera que, la prestación del servicio es regular y deficiente, teniendo en cuenta que es un oficio desorganizado inexistencia de tarifas prestablecidas por trayectos-, además, el conductor no cumple con las normas de seguridad vial, ni con los elementos de protección personal. A lo anterior se suma que, en ocasiones, los conductores muestran una actitud descortés y hasta grosera.

"Regular porque algunos son groseros, uno no le puede decir nada, a veces cobra una tarifa que no es la adecuada para un lugar que esta cerquita, o sea regular, para mi regular el servicio. Entrevista acUsuario2, Magangué.

Por su parte, algunos afirman que un transporte ilegal que no garantice la protección y seguridad del pasajero, en caso de accidente, indica que es un mal servicio. A lo anterior se suma su alto riesgo en la seguridad personal, pues el usuario está expuesto a ser víctima de actos delictivos. 
"Ese servicio es malo, (...) porque no hay seguridad, ya te dije ellos tienen un accidente y cae el pasajero ahí, y ahí lo dejan tirado, entonces, eso es un servicio completamente ilegal que ojalá lo legalizaran, pero que empresa se somete a eso, si en Cartagena hay en promedio 30 accidentes de moto diario por día". Entrevista a Transportadores 1, Arjona.

Respecto a la relación de los mototaxistas y otros conductores, se puede afirmar que está basada en una relación de carácter competitiva y colaborativa (para el caso de El Carmen de Bolívar). En el caso competitivo, la relación se tensiona por las luchas por el control del espacio, por el derecho a transportar personas y por la intolerancia vial. Estas diferencias entre las partes se manifiestan, en algunos casos, en agresiones físicas y verbales entre los mototaxistas y los otros conductores.

"La verdad es que es una relación bastante tosca se puede decir así, somos como que el agua y el aceite porque, porque hay una competencia desleal, nosotros pagamos una planilla (...) de aquí viene la seguridad social, (...) se considera que es una guerra entre comillas, pero muy desleal, desleal porque ellos tienen que pensar únicamente que tienen que echarle a los $\$ 5000$ de gasolina y lo que hagan de la tarifa y lo de ellos, mientras que esto acá tiene que trabajar para ver qué le queda, hay una libertad enorme (...) Entrevista Transportador4, Turbaco.

En El Carmen de Bolívar se indica la existencia de una relación colaborativa, puesto que los mototaxistas facilitan el trasbordo de los pasajeros que viajan en los buses o busetas intermunicipales.

“(..) se ayudan entre sí, siempre están como uno complementando al otro. (...) por ejemplo, aquí hay un transporte que es rápido El Carmen a veces deja a pasajero en la santa y ahí mismo se parquean las motos y el pasajero que se va bajando, va cogiendo su mototaxi para llegar a su destino de final, entonces complementa como de una otra manera." Entrevista Usuario8, Carmen de Bolívar.

\section{Efectos en el mantenimiento orden público}

Las manifestaciones públicas por parte de los mototaxistas han sido de carácter coyuntural, en respuesta a las acciones implementadas por las autoridades locales para regular la movilidad vial, tales como el pico y placa, la circulación de moto por sectores delimitados y el viernes sin moto. Otras razones para sus movilizaciones han sido el control del porte de documentos requeridos para la movilidad vehicular y de elementos de protección personal, tal como lo establece la ley. Estas manifestaciones, en algunos casos, generan efectos en el orden público, teniendo en cuenta que en algunos casos van acompañados de actos vandálicos -quema de llantas, cierre las calles, paralización del tráfico vehicular- y delictivos -saqueo de almacenes-, lo que genera inseguridad en la ciudadanía. No obstante, en otras oportunidades y a través del dialogo entre las partes, se han implementado acuerdos, sin llegar a conductas de este tipo.

“(...) simplemente se hizo fue una reunión colectiva digámoslo así, donde participó el alcalde municipal, la fuerza pública y otras, otras entidades de orden municipal y se le socializo la medida esa y fue aceptada mayoritariamente y no pasó a mayores" Entrevista a Funcionarios1, Turbaco. 


\section{Efectos en la accidentalidad municipal}

En los municipios objeto de estudio, las personas que ejercen el oficio de mototaxismo, de acuerdo a los entrevistados, no utilizan elementos de protección personal -guantes, chalecos reflectores, cascos- y, en muchos casos, no tienen los documentos requeridos que garanticen una seguridad vial.

"Depende del punto de vista en que se vea, en qué sentido que, si un mototaxista no cumple con lo reglamentado por la ley que estén en su seguro de SOAT, sus elementos de protección personal, sus respectivas licencias qué es lo primordial si puede afectar si puedo aumentar la tasa de accidente y también porque hay muchos que son demasiado imprudentes" Entrevista a Usuario8, Carmen de Bolívar.

Se piensa que el bajo nivel de conocimiento que tienen quienes ejercen el oficio sobre el cumplimiento de las normas de tránsito, los lleva a cometer imprudencias mientras circulan en las vías. De hecho, algunos entrevistados afirman que, en cada accidente de tránsito ocurrido en el municipio, se ve involucrada una motocicleta; por ello, consideran este oficio aumenta la probabilidad de accidentes viales. Adicionalmente, los entrevistados manifiestan que, teniendo en cuenta que una de las características de este servicio es la rapidez, los mototaxistas viven en un afán por conseguir un usuario y llevarlo rápido a su lugar de destino, sin contemplar que su conducta incrementa las posibilidades de estar involucrados en accidentes donde esté en riesgo su vida y la del pasajero.

"Primero porque ellos siempre quieren como llegar primero que otras personas, ellos no miden las consecuencias de lo que puede traer el apuro que ellos llevan, no tienen ninguna precaución, no conocen las normas (...) del sistema nacional de tránsito y transporte, no conocen ninguna norma de seguridad, no respetan la vida de los peatones, o sea eso es lo que pasa". Entrevista a Usuario4, Arjona.

Por otra parte, aparecen otros factores externos que inciden en la accidentalidad, donde las autoridades locales tienen una participación directa, entre estos se destacan: la ausencia de señalización y semaforización vial; el mal estado de las vías; el débil control en la regulación del sobrecupo y a la edad mínima del conductor y los documentos requeridos en la movilidad vial.

“(...) si ellos no tienen un control en las vías, no hay un ente que los controle, como por ejemplo la policía de tránsito, no hay señalización o las calles están pésimas, van a ocasionar esos accidentes y un riesgo para la vida de las personas de aquí, magangueleños Entrevista a Usuario1, Magangué.

\section{Percepciones sobre efectos ambientales del oficio de mototaxismo}

En esta sección se describen las percepciones que los actores locales han construido sobre los efectos ambientales del oficio de mototaxismo, tomando como referencia las sensaciones que evoca de acuerdo a los estímulos e intereses; las transformaciones que sufre el suelo rural; y cómo estos perturban el disfrute de un ambiente sano. 


\section{Efectos de la emisión de gases de combustión de las motocicletas en la calidad del aire}

Los entrevistados afirman que la emisión de gases de combustión no es exclusiva de las motocicletas; sin embargo, reconocen que el aumento del número de motos circulando en las vías también intensifica la contaminación del aire, la cual trae consecuencias a su salud, específicamente, problemas respiratorios. Como contraparte a lo anterior, algunos entrevistados expresaron que la emisión de gases de las motos es muy mínima, por lo tanto, no representa efectos negativos en la calidad del aire en los municipios.

"Bueno estos vehículos no emiten tanta combustión, por lo tanto, (...) aquí hay bastante motocicleta, pero el impacto no lo dan ellos, el impacto es por otros vehículos que utilizan un sistema de combustibles en donde si están afectando el medio ambiente, pero como tal las motocicletas no están produciendo ningún tipo de afectación (...)". Entrevista a NoUsuario2A, Arjona.

En la zona rural, de acuerdo con algunos entrevistados, la arborización actúa como protector y garante de un aire puro, de esta forma controla su contaminación.

"Hay algunas motos sí que sí digamos contamina, pero digamos que acá gracias a Dios contamos con bastante arborización digamos que tanto adentro del pueblo como afuera y entonces eso se disipa un poco." Entrevista a Usuario10, Carmen de Bolívar.

\section{Efectos de la circulación de motos en la conservación del suelo rural}

La mayoría de los entrevistados afirma que no hay efectos negativos en el uso de las motocicletas en la zona rural, porque el peso del vehículo es liviano; sin embargo, algunos afirmaron que éstas deterioran los caminos y rutas que tradicionalmente eran utilizadas por los pobladores que iban acompañados de las bestias (mulas, burros y caballos). Y finalmente, algunos entrevistados identificaron la erosión del suelo, el cual trae consigo la reducción de la fertilidad y la perdida de vegetación.

"Claro, tú sabes que es por lo menos que ellos entren el mototaxismo en una zona donde no haya pavimentación, la erosión de suelo cada vez que pasa constantemente una moto ya ese suelo no te brota más, una planta, no hay fauna, no hay forestación, esa tierra se va quitando los nutrientes que tiene, si afecta, tú no ves como en los caminos no te le sale ni una planta donde está el pasar y el pasar de un carro por qué eso afecta el suelo. Entrevista a Usuario8, Magangué.

\section{Efectos de la circulación de motos en la audición de la población}

Aun cuando la contaminación auditiva no es exclusiva de las motocicletas: los buses, carros entre otros, también contribuyen a la generación de ruido, la sensibilidad al ruido que producen las motocicletas ha intervenido en la vida diaria de la población. De acuerdo con los entrevistados, dicho ruido limita disfrutar de actividades cotidianas como conversar, ver televisión, dormir, entre otras, y adicionalmente, los sitúa en una condición de riesgo de contraer problemas auditivos.

“(...) en mi caso, cuando uno está aquí viendo televisión esas motos no dejan escuchar, entonces uno ya tiene que estar esforzando para poder escuchar, por ejemplo, la 
televisión o para escuchar lo que aquí hablamos y eso y de alguna manera eso poco a poco va a afectarle a uno la audición (...)". Entrevista a Usuario6, Turbaco.

Los mototaxistas, en algunos casos, modifican sus vehículos para aumentar el ruido al momento de acelerar. Lo anterior provoca en la población sentimientos de miedo, nervios, intranquilidad, angustia, desesperación, perdida de concentración; así como cambios en el estado de ánimo de la población, generando irritabilidad, cambio de humor, molestia, fastidio, entre otros.

"Sí las afecta, aquí particularmente cuando uno sale va viendo las esquinas porque uno siente que le pasa la moto a toda velocidad por el lado, uno vive en constante estado de alerta por el ruido que produce tantas motos". Entrevista a Usuario2, Arjona.

Al problema de contaminación sonora, se le suma el desconocimiento de los mototaxistas sobre los decibeles permisibles de acuerdo a la intensidad, duración y frecuencia del ruido en el contexto municipal. La naturalización de los ruidos provocados por la circulación de las motocicletas, muestra que cada día la población va tolerando altos niveles de decibeles que, en el mediano y largo plazo, puede tener implicaciones en la salud.

“(...) ya nos hemos aquí acostumbrado a ese ruido aquí, pero ya yo no le prestó atención a eso, nosotros no, de pronto habrá algunas personas que para ellos será estresante, pero aquí no, nos hemos familiarizado con eso, la mente se ha acostumbrado a ese ruido, así es que no hay problema". Entrevista a Usuario1, Turbaco.

\section{Efectos de la presencia de motocicletas en el atractivo visual del municipio}

La presencia de las motocicletas en los espacios públicos de los municipios se ve "feo", de acuerdo con los entrevistados, y refleja caos, desorden, desigualdades sociales, informalidad, inseguridad. En este sentido, visibiliza la situación actual de los municipios, en materia de problemas y necesidades sociales que requieren intervención del Estado.

“(...) genera es una sensación de inseguridad, de caos, en ciertas partes de nuestro municipio muestra la percepción de que no hay fuentes de empleo, que no hay fuentes de dónde se puedan adquirir educación formalizada que permita luego vinculares laboralmente de nuestros jóvenes (...) Entrevista a Funcionario 1, Turbaco.

En contraste, algunos entrevistados afirman que la presencia de mototaxis permite transportar y orientar a los visitantes a los sitios representativos del municipio. Teniendo en cuenta que el mototaxismo es un oficio legitimado en la Costa Caribe, algunos entrevistados afirman que los visitantes ya tienen previo conocimiento de la existencia de este tipo de transporte, que hace parte de la realidad del municipio. No obstante, en algunos casos, los mototaxistas asedian a los visitantes, provocando incomodidad y malestar.

"Yo pienso que es positivo porque llegan muchas personas al pueblo que no saben para dónde van y el joven del mototaxi (...) la lleva a tal parte, entonces eso les beneficia porque uno llega a una parte donde uno no conoce y tenga un medio como 
llegar así tan fácil (...) y eso es productivo hasta para el pueblo porque dicen: "no, yo sí voy al Carmen porque allá en el Carmen uno consigue todas las direcciones, porque cualquier mototaxi me lleva para donde yo quiera" Entrevista a Usuario2, Carmen de Bolívar.

\section{Percepciones sobre la efectividad de las acciones para el control del mototaxismo.}

La mayoría de los entrevistados de los municipios en estudio desconocen la existencia de medidas de regulación del oficio de mototaxi. Sin embargo, las medidas que emergieron desde discurso de los entrevistados se pueden categorizar en dos: de carácter restrictivo y de carácter pedagógico.

\section{Medidas Restrictivas}

Entre las medidas de carácter restrictivo sobresalen el pico y placa -que consiste en la restricción de la movilidad vehicular, de acuerdo a los números de placa del vehículo-; el acceso restringido a determinados sectores municipales -porque corresponde a espacio público, o porque son de uso peatonal exclusivo-, la verificación de la tenencia, al día, de documentos requeridos para la movilidad vial; y las restricciones transitorias de movilidad durante eventos y festividad.

Lo que veo es el pico y placa que es la que están utilizando ahora para regular porque hay motos que tienen pico y placa y ese día entonces ya no pueden salir y ya generan menos congestión". Entrevista a Usuario4, Magangué.

"Eh, en estos momentos tenemos sectores estacionales, o sea donde no se pueden estacionar un ejemplo es aquí al frente de la calle principal (pero solo para los mototaxistas o para todos los transportes), he, para todos, para todos y como ya te dijimos que aquí el $90 \%$ es moto, entonces es más para ellos" Grupo Focal a Funcionarios, Magangué.

\section{Medidas pedagógicas}

Los funcionarios entrevistados en los municipios de Arjona y El Carmen de Bolívar afirman que se han venido implementando medidas de carácter pedagógico. En Arjona se están desarrollando procesos con los jóvenes que se dedican a este oficio, con fin de que reconstruyan y orienten sus proyectos de vida hacia otras oportunidades profesionales y laborales. Para el caso de El Carmen de Bolívar señalan, por su parte, se están llevando a cabo campañas de sensibilización sobre el cumplimiento de las señales de tránsito y la utilización de los elementos de protección personal, dirigidas a las personas que ejercen este oficio, a fin de prevenir accidentes viales, que son muy frecuentes en el municipio.

Respecto a la valoración de la efectividad de las medidas -restrictivas y pedagógicassobresale que, en el caso de Magangué, la medida de pico y placa ha permitido aliviar la congestión vehicular; sin embargo, ha traído consigo que las personas adquieran dos motos o más -aprovechando las facilidades dadas por los distribuidores locales-, con la finalidad de no ver afectado el ejercicio de su oficio. Adicionalmente, los controles al cumplimiento de este tipo de medidas por parte de las secretarias de tránsito municipales suelen ser de carácter transitorio, lo que no garantiza que lo mototaxistas acaten esta directriz. 
“(..) lo empiezan hacer en un tiempo, por lo menos ahora en diciembre, lo que es el tránsito pone lo que es el pico y placa ya empiezan a cumplirlo por 1 por 2 por 3 días y ya después la gente hace lo que se le da la gana entonces hay un problema y es que la gente no respeta esas reglas y el tránsito no hace respetar esas reglas (...), entonces (...) no hay un orden" Entrevista a Usuario 7, Magangué

Para el caso de Turbaco, de acuerdo con algunos entrevistados, la medida del pico y placa es inviable, pues la cercanía con Cartagena genera que, cuando la medida de pico y placa se aplica en la capital bolivarense, las personas que ejercen el oficio allá se trasladan a Turbaco.

"Bueno cuando allá en Cartagena es pico y placa, los de allá se vienen para acá (...), entonces cuando aquí trabajan todos los días y allá hacen el día sin moto, los de allá se vienen para acá, hacer también desastre acá". Entrevista a Usuario 3, Turbaco

Con relación al control de documentación requerida para la movilidad de vial de las motos, se considera que es insuficiente, teniendo en cuenta que los municipios no cuentan con el personal operativo necesario para garantizar el cumplimiento de esta medida. En este sentido, las medidas restrictivas de acuerdo a los entrevistados no son cumplidas a cabalidad; para algunos, esto obedece a la contradicción en el control de este oficio, reflejado en la incoherencia de una reglamentación sobre una actividad que, en su propia naturaleza, es ilegal.

“(...) es que tú no puedes implementar regla en algo que no has legalizado, para poner reglas, tú tienes que legalizar, eso es una regla que como le vas a exigir a un mototaxista que la cumple, si es que no tienen, no le han puesto una norma que diga tú no puedes incumplir esto, tú no puedes incumplir aquello, el gobierno primero tiene que legalizar buscar una medida, buscar la manera de como los organiza para poderle exigir (...). Entrevista a Usuario8, Magangué.

\section{CONCLUSIONES}

Los resultados arrojaron que el oficio de mototaxismo surge como un medio de transporte informal que moviliza a la población de un lugar de partida a un lugar de destino, en aquellos sectores donde no opera transporte publico formal, o se dificulta su acceso; asimismo, entre las principales condiciones que conllevan a los actores locales a usar este servicio sobresale su condición de puerta a puerta, así como la rapidez y el bajo costo, en comparación con el transporte público formal convencional -buses, busetas, taxis, entre otros-. Estas cualidades del servicio generan conflictos sobre quién o quienes pueden transportar personas, teniendo en cuenta que se ha legitimado una práctica ilegal que genera unas consecuencias al servicio de transporte legal. No deja de percibirse entre los habitantes, sin embargo, la asociación del oficio con actos delictivos que atentan contra la seguridad y el bienestar de las personas, y que han llevado a la pérdida de confianza en el otro.

En cuanto a los efectos económicos del mototaxismo en el transporte formal, si bien refleja riesgos en la movilidad vial, ha facilitado el acceso de los usuarios a este tipo de transporte, dado que los mototaxis funcionan como intermediarios para el trasbordo de pasajeros desde lugar de residencia -o partida hacia los buses municipales-. No 
obstante, la llegada del mototaxismo ha remplazado el papel de los colectivos en jeeps o camionetas y las bicitaxis, hasta reducir sustancialmente su presencia. Con respecto a los productores y comerciantes, ha facilitado el transporte de carga, y en cuanto los vendedores ambulantes, se ha mantenido una relación de mutuo beneficio, por cuanto quienes ejercen el oficio también son sus clientes.

Por su parte, el oficio de mototaxismo tiene efectos en la calidad de vida de quienes los ejercen, así como en la convivencia y seguridad ciudadana. De hecho, se considera que este oficio limita la libertad humana, en tanto se ha asociado con actos delictivos que atentan contra la seguridad y el bienestar de las personas, y que han llevado a la pérdida de confianza en el otro. También puede afirmarse que ha traído consigo efectos ambientales, reflejados en la calidad del aire, conservación del suelo rural, en el atractivo visual del municipio y en la audición de la población. Respecto a las medidas de regulación de oficio del mototaxismo en los municipios en estudio, se infiere un bajo conocimiento de ellas. Entre quienes indican conocerlas, consideran son poco efectivas, puesto que son transitorias y, en algunos casos, carecen de planificación.

En general, la dinámica territorial de los municipios objeto de estudio han sufrido transformaciones, a causa principalmente de la instauración del oficio de mototaxismo, como estrategia de subsistencia en un contexto desigual, generando cambios en las relaciones socioculturales y económicas de los actores locales entre sí, y entre éstos y el territorio. De esta forma, la configuración de las percepciones que, sobre los efectos económicos, sociales y ambientales del oficio del mototaxismo, han construido los actores locales son el resultado de las experiencias sensoriales, pero también del acervo de conocimientos-culturales, ideológicos, espirituales- que han aprehendido durante su desarrollo social y que les posibilitan la apropiación de su entorno.

\section{REFERENCIAS BIBLIOGRAFICAS}

Alcaldía Municipal de Arjona. (2016). Plan de Desarrollo Municipal de Barranco de Loba 2016-2019: “Arjona Incluyente y Solidaria". Alcaldía Municipal de Arjona.

Alcaldía Municipal de Magangué. (2016). Plan de Desarrollo Municipal de Magangué 206-2019: "Magangué si avanza" (p. 206). Alcaldía Municipal de Magangué.

Alcaldía Municipal de Turbaco. (2016). Plan de Desarrollo de Turbaco 2016-2019: "Hagamos de Turbaco la ciudad que queremos y nos merecemos". Alcaldía Municipal de Turbaco.

Alcaldía Municipal El Carmen de Bolivar. (2016). Plan de Desarrollo Municipal de El Carmen de Bolívar 20162019: "Puro Pueblo: Un Gobierno con Equidad hacia la Paz." Alcaldía Municipal El Carmen de Bolivar.

Aldana Oyola, M. M. (2013). Trastornos musculoesqueleticos en mototaxistas ubicados en la zona centro de Sincelejo sucre año 2013. Aldana, 1(1), 21-23.

Angulo Salazar, R. C., \& Diaz, Y. (2011). Índice de Pobreza Multidimensional para Colombia (IPM-Colombia) 1991-2010 (No. 382; Archivos de Economía).

Arias Castilla, C. A. (2006). Enfoques teóricos sobre la percepción que tienen las personas. Horizontes Pedagógicos, 8(1), 9-22.

Avogbe, P. H., Ayi-fanou, L., Cachon, B., Chabi, N., Debende, A., Dewaele, D., Aissi, F., Cazier, F., \& Sanni, A. (2011). Hematological changes among Beninese motor-bike taxi drivers exposed to benzene by urban air pollution. African Journal of Environmental Science and Technology, 5(7), 464-472.

Barbosa, K. G. N., Lucas-Neto, A., Gama, B. D., Lima-Neto, J. C., Lucas, R. S. C. C., \& D'Ávila, S. (2014). Injuries and absenteeism among motorcycle taxi drivers who are victims of traffic accidents. Journal of Forensic and Legal Medicine, 26, 15-18. 
Brieva Paternina, J. L., Tinoco Cantillo, U. A., Restrepo Herrera, J. F., \& Arango Buelvas, L. J. (2011). El mototaxismo en Sincelejo, un análisis socioeconómico.

Burbano, A. M. (2009). La convivencia ciudadana: su análisis a partir del "aprendizaje por reglas\&quot; Revista Colombiana de Educación, 57, 28-45.

Castillo Avila, I., Galarza Herrera, B., \& Palomino Gómez, H. (2013). Condiciones de trabajo y salud de mototaxistas Cartagena - Colombia Work and healht conditions in motorcycle taxi drivers Cartagena - Colombia. Salud Uninorte, 29(3), 514-524.

Castillo Osorio, B. (2010). El mototaxismo ¿problema informal o un medio de generación de ingresos para resolver en parte el problema del desempleo en Montería? Económicas CUC, 31(31), 89-104.

Chavis, C., \& Daganzo, C. F. (2013). Analyzing the structure of informal transit: The evening commute problem. Research in Transportation Economics, 39(1), 277-284.

Cini, M. A., Prado, B. G., Hinnig, P. D. F., Fukushima, W. Y., \& Adami, F. (2014). Influence of type of helmet on facial trauma in motorcycle accidents. British Journal of Oral and Maxillofacial Surgery.

Escobar, J., \& Bonilla Jimenez, I. (2009). Grupos Focales: una guía conceptual y metodológica. Cuadernos Hispanoamericanos de Psicología, 9(1), 51-67.

Espinosa, C., Rodriguez, C., \& Uribe, maria carolina. (2008). El impacto socieconómico del fenomeno del mototaxismo en montería. 226.

González Galán, E. (2008). Percepción y usos de espacios públicos madrileños. Universidad Complutense de Madrid.

Guillen, M. D. V, \& Ishida, H. (2004). Motorcycle-propelled public transport and local policy development: the case of "tricycles" and "habal-habal" in Davao city Philippines. IATSS Research, 28(1), 56-66.

Hagen, J. X., Pardo, C. F., \& Valente, J. B. (2016). Motivations for motorcycle use for Urban travel in Latin America: A qualitative study. Transport Policy, 49, 93-104.

Herazo B., Y., Domínguez A., R., Olarte B., S., \& Quitian O., L. (2011). Discapacidad y mototaxismo en Cartagena, Colombia. (Spanish). Disability and Motorcycle Taxi Drivers in Cartagena, Colombia. (English), 29, 469-473.

Hinestroza-filigrana, M. L., \& Toro-mayor, M. L. (2015). Condiciones de Salud y Trabajo de los Mototaxistas en Palmira, Colombia (2014). 5(1), $19-26$.

Jimenez, A., Bocarejo, J. P., Zarama, R., \& Yerpez, J. (2015). A case study analysis to examine motorcycle crashes in Bogota, Colombia. Journal of Safety Research, 52, 29-38.

Maza Ávila, F. J., Daniels Puello, A., Blanco Bello, R., \& Vergara Schmalbach, J. C. (2017). El transporte informal en los municipios de Arjona, Turbaco, El Carmen de Bolívar y Magangué, departamento de Bolívar (p. 125).

Maza Avila, F. J., \& Perez Gonzalez, M. del C. (2015). Pertinencia de la oferta educativa de la Zodes Montes de María desde sus capacidades productivas y económicas. Palobra, Palabra Que Obra, 15.

Ojeda, M. G. (2005). Seguridad Ciudadana邓: El Aporte de las Metodologías Implicativas Citizen Security: A contribution of Implacative and Participative Methodologies. XIV, 63-78.

Rubio, M. J., \& Varas, J. (2004). Ámbitos de análisis de la realidad. El análisis de la realidad en la intervención social. Métodos y técnicas de investigación. Editorial CCS. Madrid, 105-124.

Sandoval Casilimas, C. (1996). Investigación cualitativa. In Módulo. https://doi.org/958-9329-18-7

Simon, R., Barnes, W., Mohammad, A., Sevak, S., Ziegler, K., Iacco, A., \& Anczyk, R. (2016). Effects of repealing the motorcycle helmet law in Michigan. The American Journal of Surgery, 214(3), 407-412.

Vargas Melgarejo, L. M. (1994). Sobre el concepto de percepción. Alteridades, 4(8), 47-53. 